\title{
Traitement d'eaux usées urbaines par infiltration-percolation sur sable et sur substrat de coco après un bassin anaérobie de lagune sous climat tropical \\ Urban wastewater treatment by infiltration-percolation with sand and coconut media after an anaerobic pond under tropical climate
}

\author{
Martine Kone, Lucien Bonou, Jean Koulidiati, Pierre Joly, Soumaïla Sodre et \\ Yvette Bouvet
}

Volume 25, numéro 2, 2012

Reçu le 29 juin 2010, accepté le 29 avril 2012

URI : https://id.erudit.org/iderudit/1011604ar

DOI : https://doi.org/10.7202/1011604ar

\section{Aller au sommaire du numéro}

Éditeur(s)

Université du Québec - INRS-Eau, Terre et Environnement (INRS-ETE)

ISSN

1718-8598 (numérique)

Découvrir la revue

Citer cet article

Kone, M., Bonou, L., Koulidiati, J., Joly, P., Sodre, S. \& Bouvet, Y. (2012). Traitement d'eaux usées urbaines par infiltration-percolation sur sable et sur substrat de coco après un bassin anaérobie de lagune sous climat tropical. Revue des sciences de l'eau / Journal of Water Science, 25(2), 139-151. https://doi.org/10.7202/1011604ar
Résumé de l'article

Au Burkina Faso, l'un des soucis majeurs demeure la gestion des eaux résiduaires dans les villes. Notre objectif a été d'étudier des procédés biologiques alternatifs destinés aux petites et moyennes collectivités et particulièrement adaptés aux conditions climatiques tropicales. Nous avons examiné les performances épuratoires de deux matériaux de filtration que sont le sable et un substrat de coco concassé (fibres de coco), sur des systèmes pilotes recevant des effluents issus d'un bassin anaérobie de lagunage. Les résultats montrent des performances croissantes de $93 \%$ à $95 \%$ sur la DCO (Demande Chimique en Oxygène) et un abattement moyen sur la DBO (Demande Biochimique en Oxygène) atteignant $99 \%$, avec la colonisation progressive du massif de sable par la biomasse. Avec le substrat de coco les rendements moyens sur la DCO passent successivement de $15 \%$ à $51 \%$ lorsque

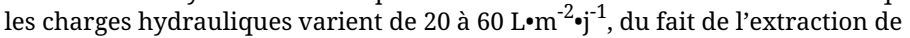
composés phénoliques du substrat. Cependant, il présente la même efficacité que le sable pour l'élimination de la pollution biodégradable.

Les rendements en azote Kjeldahl sont de $75 \%$ et $84 \%$ pour le substrat de coco et le sable respectivement, alors que le filtrat issu du substrat de coco n'a donné que $7 \mathrm{mg} \cdot \mathrm{L}^{-1}$ en moyenne de nitrates. Dans le même temps, les concentrations en nitrates atteignent $40 \mathrm{mg} \mathrm{L}^{-1}$ pour le massif de sable. Ce comportement du substrat de coco pourrait être attribué à la formation de complexes entre composés azotés et les tanins issus de ce matériau. 


\section{TRAITEMENT D'EAUX USÉES URBAINES PAR INFILTRATION- PERCOLATION SUR SABLE ET SUR SUBSTRAT DE COCO APRÈS UN BASSIN ANAÉROBIE DE LAGUNAGE SOUS CLIMAT TROPICAL}

Urban wastewater treatment by infiltration-percolation with sand and coconut media after an anaerobic pond under tropical climate

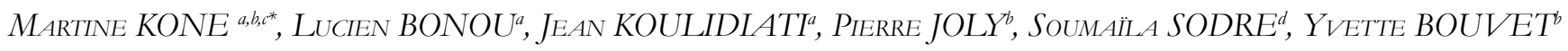

${ }^{a}$ UFR/SEA, Université de Ouagadougou, BP 7021, Ouagadougou 03, Burkina Faso.

bLaboratoire d'Écologie des Hydrosystèmes Naturels et Anthropisés, Université Claude Bernard Lyon 1, 43, boulevard du 11 nov 1918, 69622 Villeurbanne, France.

'Institut de Recherche en Sciences Appliquées et Technologies / CNRST, 03 BP 7047, Ouagadougou 03, Burkina Faso.

dOffice National de l'Eau et de l'Assainissement (ONEA), 01 BP 170, Ouagadougou 01, Burkina Faso.

Reçu le 29 juin 2010, accepté le 29 avril 2012

\section{RÉSUMÉ}

Au Burkina Faso, l'un des soucis majeurs demeure la gestion des eaux résiduaires dans les villes. Notre objectif a été d'étudier des procédés biologiques alternatifs destinés aux petites et moyennes collectivités et particulièrement adaptés aux conditions climatiques tropicales. Nous avons examiné les performances épuratoires de deux matériaux de filtration que sont le sable et un substrat de coco concassé (fibres de coco), sur des systèmes pilotes recevant des effluents issus d'un bassin anaérobie de lagunage. Les résultats montrent des performances croissantes de $93 \%$ à $95 \%$ sur la DCO (Demande Chimique en Oxygène) et un abattement moyen sur la $\mathrm{DBO}_{5}$ (Demande Biochimique en Oxygène) atteignant $99 \%$, avec la colonisation progressive du massif de sable par la biomasse. Avec le substrat de coco les rendements moyens sur la DCO passent successivement de $15 \%$ à $51 \%$ lorsque les charges hydrauliques varient de 20 à $60 \mathrm{~L} \cdot \mathrm{m}^{-2} \bullet \mathrm{j}^{-1}$, du fait de l'extraction de composés phénoliques du substrat. Cependant, il présente la même efficacité que le sable pour l'élimination de la pollution biodégradable.

Les rendements en azote Kjeldahl sont de $75 \%$ et $84 \%$ pour le substrat de coco et le sable respectivement, alors que le filtrat issu du substrat de coco n'a donné que $7 \mathrm{mg}^{\bullet} \mathrm{L}^{-1}$ en moyenne de nitrates. Dans le même temps, les concentrations en nitrates atteignent $40 \mathrm{mg} \cdot \mathrm{L}^{-1}$ pour le massif de sable. Ce comportement du substrat de coco pourrait être attribué à la formation de complexes entre composés azotés et les tanins issus de ce matériau.

Mots clés : Sable, substrat de coco, eaux usées, infiltration, nitrification.

Auteur pour correspondance :

Téléphone: +22670307668/ +22650356031

Télécopieur : +22650357029

Courriel : koneba2003@yahoo.fr 


\section{ABSTRACT}

In Burkina Faso, one of the major problems is the management of waste water in the cities. Biological processes for the small and middle-size communities exist, for example the infiltration-percolation approach that is particularly adapted to tropical climatic conditions. The efficiency of two filtration media (sand and coconut fiber) was tested in pilot systems downstream from an anaerobic pond acting as a primary treatment. The results show increasing efficiencies of COD removal, from $93 \%$ to $95 \%$, and $\mathrm{BOD}_{5}$ removal efficiency reaches $99 \%$, with the progressive colonization of the sand filter by the biomass. The filtration media composed of crushed coconut exhibited COD removal efficiencies of $15 \%$ and $51 \%$ for low $\left(20 \mathrm{~L}^{\bullet} \mathrm{m}^{-2} \bullet \mathrm{d}^{-1}\right)$ and high $\left(60 \mathrm{~L} \cdot \mathrm{m}^{-2} \bullet \mathrm{d}^{-1}\right)$ hydraulic loads respectively, because of the progressive extraction of phenolic compounds from the coconut material during the first leachings. However, the coconut media gave almost the same efficiency as the sand with respect to the removal of biodegradable pollution.

Nitrogen removal was also investigated, and both media transformed Kjeldahl nitrogen efficiently (75\% and 84\%). However, the coconut media gave the lowest concentration of nitrate $\left(7 \mathrm{mg}^{\bullet} \mathrm{L}^{-1}\right)$ because of tannins. The nitrate concentrations in the effluent of the sand filters reached $40 \mathrm{mg} \cdot \mathrm{L}^{-1}$. This behavior of coconut media can be attributed to complexes formed between nitrogenous compounds and tannins coming from this material.

Keywords: Sand, coconut material, waste water, infiltration, nitrification.

\section{INTRODUCTION}

La démographie galopante en milieu urbain non accompagnée d'un plan conséquent d'urbanisation, conjuguée avec le manque d'ouvrages d'assainissement adaptés, sont les causes de déversements d'eaux usées et excréta dans la plupart des grandes agglomérations africaines. Le Burkina Faso, à l'instar de nombre de pays en développement, est confronté à des problèmes d'assainissement.

En effet, les fosses septiques, les latrines VIP (Ventilated Improved Pit) et les puisards proposés en assainissement autonome n'assurent pas un traitement suffisant des eaux usées et excréta, et génèrent des matières de vidange dont l'absence de gestion est source de pollution de l'environnement et des ressources en eaux superficielles et souterraines (KONE et al., 2009; TILLEY et al., 2008; WETHE et al., 2003) avec, pour conséquence, une persistance des maladies hydriques telles que les diarrhées, la fièvre typhoïde, le choléra, etc. Les lits d'infiltration-percolation constituent une alternative en assainissement non collectif, aussi bien en milieu rural qu'en milieu urbain. C'est dans cette optique que nous avons expérimenté le traitement d'eaux usées chargées comme celles reçues par la station d'épuration de Ouagadougou.

Nous avons choisi d'installer un pilote d'infiltrationpercolation sur sable, utilisant du sable de rivière pour sa capacité d'élimination de la DCO (WANKO et al., 2005) et un pilote d'infiltration-percolation sur substrat de coco concassé, tous deux alimentés par un effluent sortant d'un bassin anaérobie de lagunage qui fait office de traitement primaire.

Ce sont des filières peu expérimentées en Afrique sous climat tropical, ce qui justifie de s'intéresser aux performances épuratoires d'une succession de bassins anaérobies et lits d'infiltration-percolation même si, par ailleurs, un certain nombre d'études menées sur ce procédé de traitement des eaux usées et boues de vidange ont fait la preuve de l'efficacité des lits d'infiltration-percolation sur sable (COULIBALY et al., 2008; GNAGNE et BRISSAUD, 2003; KOUAME et al., 2008; YEO et al., 2008).

Pourquoi le choix du substrat de coco? La fibre de coco présente des caractéristiques physiques intéressantes qui pourraient en faire un bon support de filtration en traitement des eaux usées. Elle est quasi imputrescible grâce à une forte teneur en lignine et un rapport $\mathrm{C} / \mathrm{N}$ important qui lui confère une grande stabilité structurale (FALIENOR, 2006).

C'est un matériau qui connaît un engouement croissant à travers le monde dans le domaine de l'assainissement autonome et les pays côtiers, voisins du Burkina Faso dont la Côte d'Ivoire, inondent le pays de noix de coco. Il a donc semblé intéressant de tester les capacités de traitement des eaux usées avec ce matériau dans les conditions d'un pays sahélien à climat tropical, ce qui serait aussi une façon de valoriser ce sous-produit issu du commerce des noix de coco.

L'objet de ce travail est de mettre en exergue les performances comparées de deux supports de filtration en matière d'abattement sur la DCO et la $\mathrm{DBO}_{5}$, mais également de comparer leurs capacités de nitrification et d'abattement sur l'azote réduit dans des conditions de charges hydrauliques croissantes. 


\section{MATÉRIEL ET MÉTHODES}

\subsection{Dispositifs expérimentaux}

Les dispositifs expérimentaux sont composés de deux fûts cylindriques d'une capacité de $250 \mathrm{~L}$, avec un diamètre intérieur de $0,54 \mathrm{~m}$, et une hauteur de $1 \mathrm{~m}$ (Figure 1). Une cheminée d'aération, soit un tube en PVC de $90 \mathrm{~cm}$ de longueur et de $8 \mathrm{~cm}$ de diamètre, est placée au centre du massif, au-dessus de la couche de drainage.

La composition des massifs se présente comme suit : de bas en haut $10 \mathrm{~cm}$ de granite de diamètre 10/25 mm (couche de drainage), $10 \mathrm{~cm}$ de granite $3 / 8 \mathrm{~mm}$ (couche de transition) et $65 \mathrm{~cm}$ de sable moyen ou de substrat de coco (couche de filtration).

Les fûts inclinés de $10 \%$ par rapport au plan horizontal présentent des trous de $5 \mathrm{~mm}$ de diamètre sur le quart du fond dans le sens de l'inclinaison, servant de sortie pour les eaux traitées.

Le sable, de granulométrie moyenne, présente les caractéristiques suivantes: $\mathrm{D}_{10}=0,30 \mathrm{~mm}, \mathrm{D}_{60}=0,80 \mathrm{~mm}$, soit $\mathrm{D}_{60} / \mathrm{D}_{10}=2,66$ et $0,6 \%$ de fines (de diamètre inférieur à $80 \mu \mathrm{m}$ ). Le substrat de coco, issu de la coque entière de coco(enveloppe et bourre) réduit en morceaux de $5 \mathrm{~mm}$ en moyenne, a été macéré dans de l'eau renouvelée régulièrement pendant un mois, afin d'extraire les sels qui donnent une conductivité très élevée au filtrat. Cette salinité est liée au fait que les cocotiers sont plantés au bord de la mer (FALIENOR, 2006). De plus, des composés phénoliques, dont les tanins en particulier, contenus dans le substrat de coco colorent intensément le filtrat ou l'eau de macération. À l'issue de cette phase de macération, les massifs constitués ont été lavés à l'eau claire pendant une semaine pour réduire la teneur en particules fines.

Les pilotes sont désignés par FSA (filtre à sable aéré) et FCA (filtre à substrat de coco aéré). Le terme «brute " correspond à l'effluent d'alimentation.

\subsection{Fréquence et mode d'alimentation des pilotes}

Les trois charges hydrauliques successives suivantes ont été expérimentées : $20 \mathrm{~L} \cdot \mathrm{m}^{-2} \bullet \mathrm{j}^{-1}$ (juin et juillet 2009 ), $40 \mathrm{~L} \cdot \mathrm{m}^{-2} \bullet \mathrm{j}^{-1}$ (août à novembre 2009) et $60 \mathrm{~L} \cdot \mathrm{m}^{-2} \bullet \mathrm{j}^{-1}$ (janvier et février 2010).

Les pilotes sont alimentés dans les mêmes conditions par bâchées en deux temps avec l'effluent brut. Ces eaux d'une typologie domestique sont composées d'eaux usées domestiques issues de la ville, des rejets d'une brasserie et de

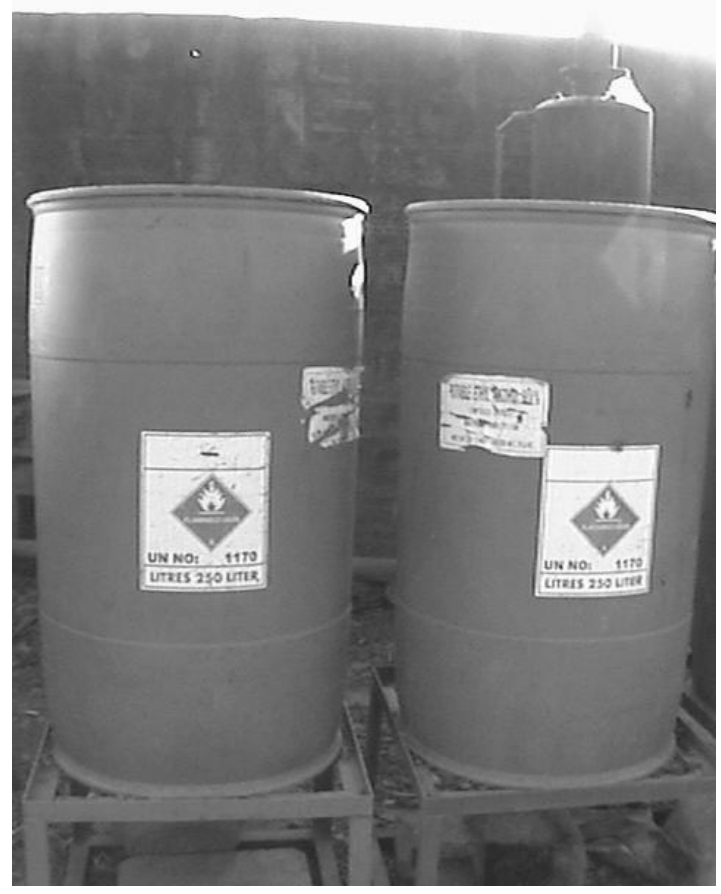

Figure 1. Vue des pilotes. 
ceux d'un abattoir. Le tableau 1 donne les caractéristiques physico-chimiques de l'effluent d'alimentation.

Pour simuler les pics de rejets d'eaux usées de la population, les pilotes sont alimentés à $8 \mathrm{~h} 00$ et $14 \mathrm{~h} 30$ les lundis, mardis et mercredis, et laissés au repos les quatre derniers jours de la semaine.

\subsection{Régime hydraulique au sein des massifs}

Les deux matériaux de filtration ayant des conductivités hydrauliques différentes, il nous a paru pertinent d'apprécier l'hydraulique des premières heures de fonctionnement d'autant plus que le ressuyage est très rapide au sein du substrat de coco. C'est ainsi que le débit moyen de percolation du troisième jour $(\mathrm{Q}=\mathrm{V} / \mathrm{t})$, correspondant au rapport des volumes recueillis toutes les $30 \mathrm{~min}(\mathrm{~V})$, au cours des trois premières heures suivant le début de l'écoulement, a été mesuré pour les trois charges hydrauliques (Tableau 2).

\subsection{Prélèvements et analyse des échantillons}

Un prélèvement quotidien a été effectué sur l'effluent d'alimentation aux fins d'analyse. Pour les deux premières charges hydrauliques, un échantillon moyen de 24 h est prélevé à la sortie des pilotes à l'issue du troisième jour d'alimentation. Pour la charge hydraulique de $60 \mathrm{~L} \cdot \mathrm{m}^{-2} \bullet \mathrm{j}^{-1}$, les prélèvements ont été quotidiens pendant les trois jours d'alimentation.

Les paramètres suivants ont été analysés : la DCO, la $\mathrm{DBO}_{5}$, les matières en suspension (MES), les nitrates $\left(\mathrm{NO}_{3}{ }^{-}\right)$, les nitrites $\left(\mathrm{NO}_{2}^{-}\right)$, l'ammonium $\left(\mathrm{NH}_{4}^{+}\right)$et l'azote Kjeldahl (NTK).

La DCO est déterminée par oxydation au dichromate de potassium en milieu acide conformément à la norme AFNOR T-90-101. La lecture de la valeur de l'absorbance est faite par spectrophotométrie à la longueur d'onde de $600 \mathrm{~nm}$ ou $420 \mathrm{~nm}$ selon la gamme de DCO choisie. $\mathrm{La} \mathrm{DBO}_{5}$ est déterminée par la méthode manométrique avec des manomètres Oxitop WTW, selon la norme NF 90-103. Les MES sont obtenues par filtration sous vide sur un filtre en microfibre de verre GF/C conformément à la norme française NF 90-105. Les concentrations en nitrates, nitrites et ammonium sont déterminées par des méthodes colorimétriques, la lecture est faite au spectrophotomètre DR/2010 de HACH LANGE. L'azote Kjeldahl est déterminé après minéralisation des matières organiques en milieu acide et en présence de catalyseur, puis entraînement à la vapeur en milieu alcalin de l'azote ammoniacal obtenu et dosage par titrimétrie conformément à la norme française NF 90-110.

Tableau 1. Valeurs caractéristiques des paramètres mesurés pour l'effluent d'alimentation issu du bassin anaérobie de lagunage à microphytes.

Table 1. Design parameters and pollutant concentrations in the anaerobic pond effluent.

\begin{tabular}{|c|c|c|c|c|c|}
\hline \multirow{2}{*}{ Paramètres } & \multirow{2}{*}{$n$} & \multicolumn{3}{|c|}{ Valeurs } & \multirow{2}{*}{ Écarts-types } \\
\hline & & Min & Max & Moyenne & \\
\hline $\mathrm{DCO}\left(\mathrm{mg} \mathrm{O}_{\left.2 \cdot \mathrm{L}^{-1}\right)}\right.$ & 47 & 659 & 1580 & 1060 & 251 \\
\hline $\mathrm{DBO}_{5}\left(\mathrm{mg} \mathrm{O}_{2} \mathrm{~L}^{-1}\right)$ & 47 & 500 & 980 & 812 & 146 \\
\hline $\mathrm{N}-\mathrm{NO}_{3}\left(\mathrm{mg} \bullet \mathrm{L}^{-1}\right)$ & 37 & 0,30 & 5 & 1,82 & 0,70 \\
\hline $\mathrm{N}-\mathrm{NH}_{4}\left(\mathrm{mg} \bullet \mathrm{L}^{-1}\right)$ & 26 & 19 & 39 & 31 & 5 \\
\hline $\mathrm{N}-\mathrm{NTK}\left(\mathrm{mg} \bullet \mathrm{L}^{-1}\right)$ & 12 & 54 & 71 & 63 & 12 \\
\hline $\operatorname{MES}\left(\mathrm{mg} \bullet \mathrm{L}^{-1}\right)$ & 12 & 138 & 227 & 187 & 34 \\
\hline
\end{tabular}

Tableau 2. Débits moyens de percolation.

Table 2. Mean percolation rates.

\begin{tabular}{|c|c|c|}
\hline $\begin{array}{c}\text { Charge hydraulique } \\
\quad\left({\left.\mathrm{L} \bullet \mathrm{m}^{-2} \bullet \mathrm{j}^{-1}\right)}^{-1}\right.\end{array}$ & $\begin{array}{c}\text { QFSA } \\
\left(\mathrm{mL} \bullet \mathrm{min}^{-1}\right)\end{array}$ & $\begin{array}{c}\text { QFCA } \\
\left(\mathrm{mL} \bullet \mathrm{min}^{-1}\right)\end{array}$ \\
\hline 20 & 1,77 & 3,00 \\
\hline 40 & 2,16 & 11,66 \\
\hline 60 & 7 & 25 \\
\hline
\end{tabular}




\section{RÉSULTATS}

\subsection{Typologie des eaux résiduaires urbaines (ERU)}

Le tableau 1 donne un aperçu des valeurs moyennes et écarts-types de l'effluent d'alimentation, à la sortie du bassin anaérobie. En dépit du séjour en bassin anaérobie, les ERU initialement très chargées présentent des valeurs assez élevées en $\mathrm{DCO}$ et $\mathrm{DBO}_{5}$ sous forme dissoute compte tenu du fait que plus de $50 \%$ des charges hydrauliques entrant à la station sont issues de la brasserie. On remarque également que le rapport des valeurs moyennes $\mathrm{DCO} / \mathrm{DBO}_{5}$ inférieur à 1,5 montre un caractère très biodégradable de l'ERU.

\subsection{Performances épuratoires des pilotes}

\subsubsection{Demande chimique en oxygène}

La figure 2 donne l'évolution de la DCO pour les trois débits successifs au niveau des deux pilotes en rapport avec la DCO de l'effluent d'alimentation. On constate une augmentation de la DCO avec le FCA les trois premières semaines après la mise en fonctionnement du pilote, avec des rendements négatifs atteignant $-27 \%$ pendant cette période pour finalement connaître une amélioration avec un abattement global sur ce paramètre de $15 \%$. Par contre, l'évolution de la DCO du FSA montre un bon abattement avec des concentrations inférieures à $100 \mathrm{mg} \bullet \mathrm{L}^{-1}$ et un rendement moyen de $93 \%$.

On note une amélioration des performances du FCA dans la deuxième phase (à partir du mois d'août), avec un abattement moyen sur la DCO de $40 \%$, tandis que le FSA assure un abattement moyen sur la DCO de $95 \%$, une performance en hausse avec la colonisation progressive du massif par les micro-organismes, et ce, en dépit de la charge hydraulique qui a doublé.

Lorsque la charge hydraulique passe à $60 \mathrm{~L} \cdot \mathrm{m}^{-2} \bullet \mathrm{j}^{-1}$ à la mi-décembre, la tendance acquise avec la précédente charge hydraulique se maintient avec un accroissement des rendements épuratoires qui sont de $51 \%$ et $95 \%$ respectivement pour le FCA et le FSA.

Globalement, le FSA demeure performant de façon croissante sur toute la durée de l'étude avec des rendements moyens atteignant $95 \%$. Le FCA montre une amélioration de ses performances, mais reste largement en deçà du FSA en matière d'abattement de la DCO.

\subsubsection{Demande biochimique en oxygène}

La figure 3 présente l'évolution temporelle de la $\mathrm{DBO}_{5}$ avec les débits successifs de 40 et $60 \mathrm{~L} \cdot \mathrm{m}^{-2} \bullet \mathrm{j}^{-1}$. On constate que les courbes traduisant l'évolution de la $\mathrm{DBO}_{5}$ au niveau du
FCA et du FSA sont pratiquement confondues. En effet, nous avons comme rendements moyens respectifs, 98 et $99 \%$ pour le FCA et le FSA avec une charge hydraulique de $40 \mathrm{~L}_{\bullet} \mathrm{m}^{-2} \bullet \mathrm{j}^{-1}$. Ces rendements se maintiennent à la charge hydraulique de $60 \mathrm{~L} \cdot \mathrm{m}^{-2} \bullet \mathrm{j}^{-1}$. La figure 4 montre les performances comparées des deux matériaux de filtration.

\subsubsection{Matières en suspension}

La figure 5 présente l'évolution dans le temps des teneurs en MES. Les concentrations en MES de l'effluent d'alimentation varie entre 137 et $226 \mathrm{mg} \bullet \mathrm{L}^{-1}$, tandis que le FSA, qui assure un abattement moyen de $91 \%$, donne des teneurs moyennes en MES de $16 \pm 15 \mathrm{mg} \bullet \mathrm{L}^{-1}$. Dans le même temps, le FCA présente des teneurs moyennes $90 \pm 59 \mathrm{mg} \bullet \mathrm{L}^{-1}$ avec un abattement moyen $52 \%$. Les plus faibles concentrations ont été obtenues au début du fonctionnement des pilotes comme l'indique la figure 5 .

\subsubsection{Azote}

Avec la charge hydraulique de $20 \mathrm{~L} \cdot \mathrm{m}^{-2} \bullet \mathbf{j}^{-1}$, nous n'avons pas pu faire la mesure de la teneur en nitrates et ammonium du filtrat issu du FCA, parce que celui-ci était très coloré, même après une dilution par un facteur 10 , alors que la méthode utilisée est basée sur la colorimétrie.

Avec le FSA, on constate une augmentation progressive des concentrations en nitrates variant entre 2,90 et 26,9 $\mathrm{mg} \mathrm{N}-\mathrm{NO}_{3} \cdot \mathrm{L}^{-1}$ dans la première phase d'expérimentation pour atteindre $30 \mathrm{mg} \mathrm{N}-\mathrm{NO}_{3} \bullet \mathrm{L}^{-1}$ dans la deuxième phase (Figure 6), et ce, pour des teneurs en azote Kjeldahl (formes oxydables de l'azote) en entrée de $63 \mathrm{mg} N T K \cdot L^{-1}$.

À $40 \mathrm{~L} \cdot \mathrm{m}^{-2} \bullet \mathrm{j}^{-1}$, le filtrat issu du pilote à substrat de coco (FCA) est beaucoup moins coloré et les analyses des nitrates ont pu être effectuées après dilution. Les teneurs en nitrates varient entre 5 et $9 \mathrm{mg} \mathrm{N}-\mathrm{NO}_{3} \cdot \mathrm{L}^{-1}$ pendant cette phase d'alimentation (Figure 6). Elles décroissent à $60 \mathrm{~L}_{\bullet} \mathrm{m}^{-2} \bullet \mathrm{j}^{-1}$ pour se maintenir à une moyenne à peine supérieure à $2 \mathrm{mg}^{\bullet} \mathrm{L}^{-1}$, valeur correspondant à la teneur en nitrates de l'effluent brut d'alimentation (Figure 7).

Cependant, on note un abattement assez significatif des teneurs en $\mathrm{NH}_{4}^{+}$et NTK avec des rendements épuratoires moyens de $75 \%$ respectivement pourNH ${ }_{4}^{+}$et NTK en dépit de la faible nitrification.

Avec le débit de $60 \mathrm{~L} \cdot \mathrm{m}^{-2} \bullet \mathrm{j}^{-1}$, on constate qu'au niveau du FSA, l'azote ammoniacal disponible dans l'effluent est entièrement nitrifié (Figure 7). En effet, les concentrations en nitrates varient entre 15 et $44 \mathrm{mg} \mathrm{N}-\mathrm{NO}_{3} \cdot \mathrm{L}^{-1}$ pour des teneurs en $\mathrm{NH}_{4}{ }^{+}$à l'entrée variant entre 19 et $39 \mathrm{mg} \mathrm{N}-\mathrm{NH}_{4} \bullet \mathrm{L}^{-1}$. De l'azote organique transformé en $\mathrm{NH}_{4}^{+}$a pu être oxydé en $\mathrm{NO}_{3}^{-}$ également puisqu'il est reconnu que $\mathrm{NH}_{4}^{+}$est en partie adsorbé au sein du massif filtrant. En outre, l'azote Kjeldahl connaît 


\begin{tabular}{|l|l|l|}
\hline DCO à $20{\mathrm{~L} \cdot \mathrm{m}^{-2} \bullet \mathrm{j}^{-1}}^{-1}$ & DCO à $40{\mathrm{~L} \bullet \mathrm{m}^{-2} \cdot \mathrm{j}^{-1}}^{-1}$ & DCO à $60 \mathrm{~L} \bullet \mathrm{m}^{-2} \bullet \mathrm{j}^{-1}$ \\
\hline
\end{tabular}

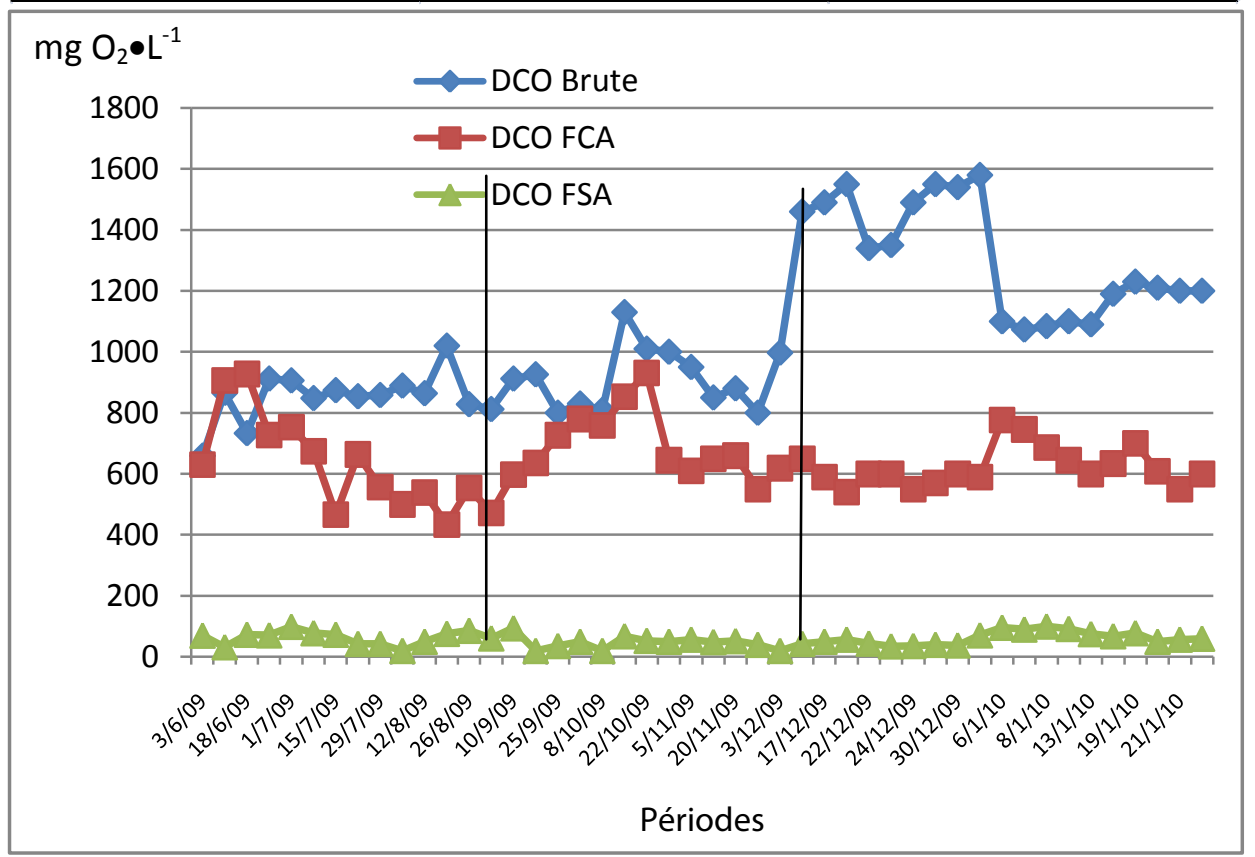

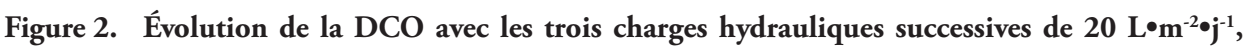
$40 \mathrm{~L}^{\circ} \mathrm{m}^{-2} \bullet \mathrm{j}^{-1}$ et $60 \mathrm{~L} \cdot \mathrm{m}^{-2} \bullet \mathrm{j}^{-1}$.

Chemical oxygen demand removal with the three successive loads of $20 \mathrm{~L}^{\bullet} \mathrm{m}^{-2} \cdot \mathrm{j}^{-1}$, $40 \mathrm{~L} \cdot \mathrm{m}^{-2} \cdot \mathrm{j}^{-1}$ and $60 \mathrm{~L} \cdot \mathrm{m}^{-2} \cdot \mathrm{j}^{-1}$.

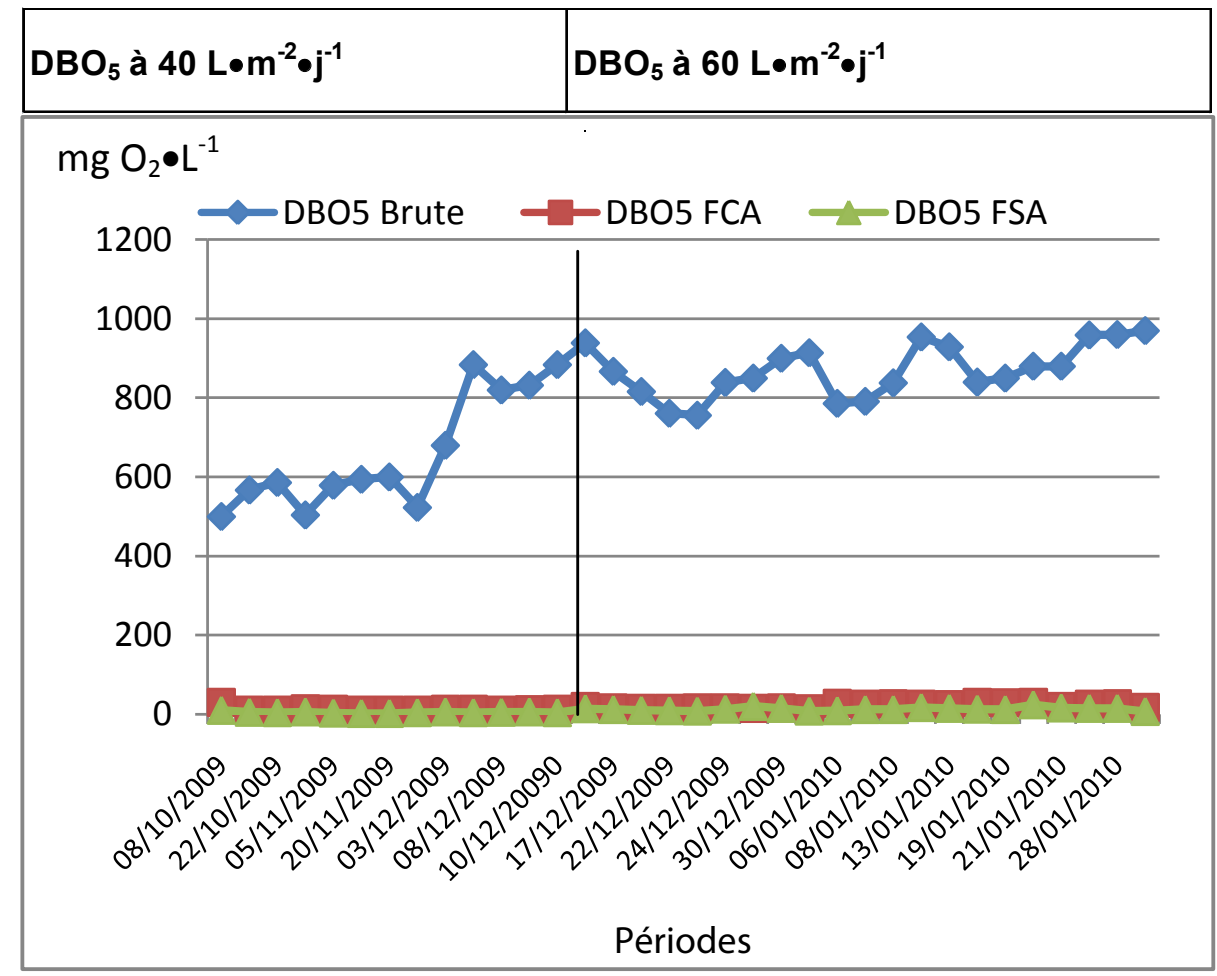

Figure 3. Évolution temporelle de $\mathrm{la}_{\mathrm{DBO}}$ du filtre à sable aéré (FSA) et du filtre à substrat

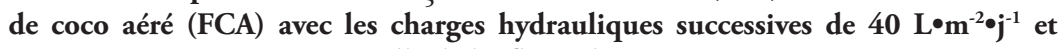
$60 \mathrm{~L}^{\bullet} \mathrm{m}^{-2} \bullet \mathrm{j}^{-1}$ en rapport avec celle de l'effluent brut.

Five-day biochemical oxygen demand $\left(B O D_{5}\right)$ removal for FSA and FCA with the two loads of $40 \mathrm{~L} \cdot \mathrm{m}^{-2} \cdot j^{-1}$ and $60 \mathrm{~L} \cdot \mathrm{m}^{-2} \cdot j^{-1}$, compared to the effluent BOD 


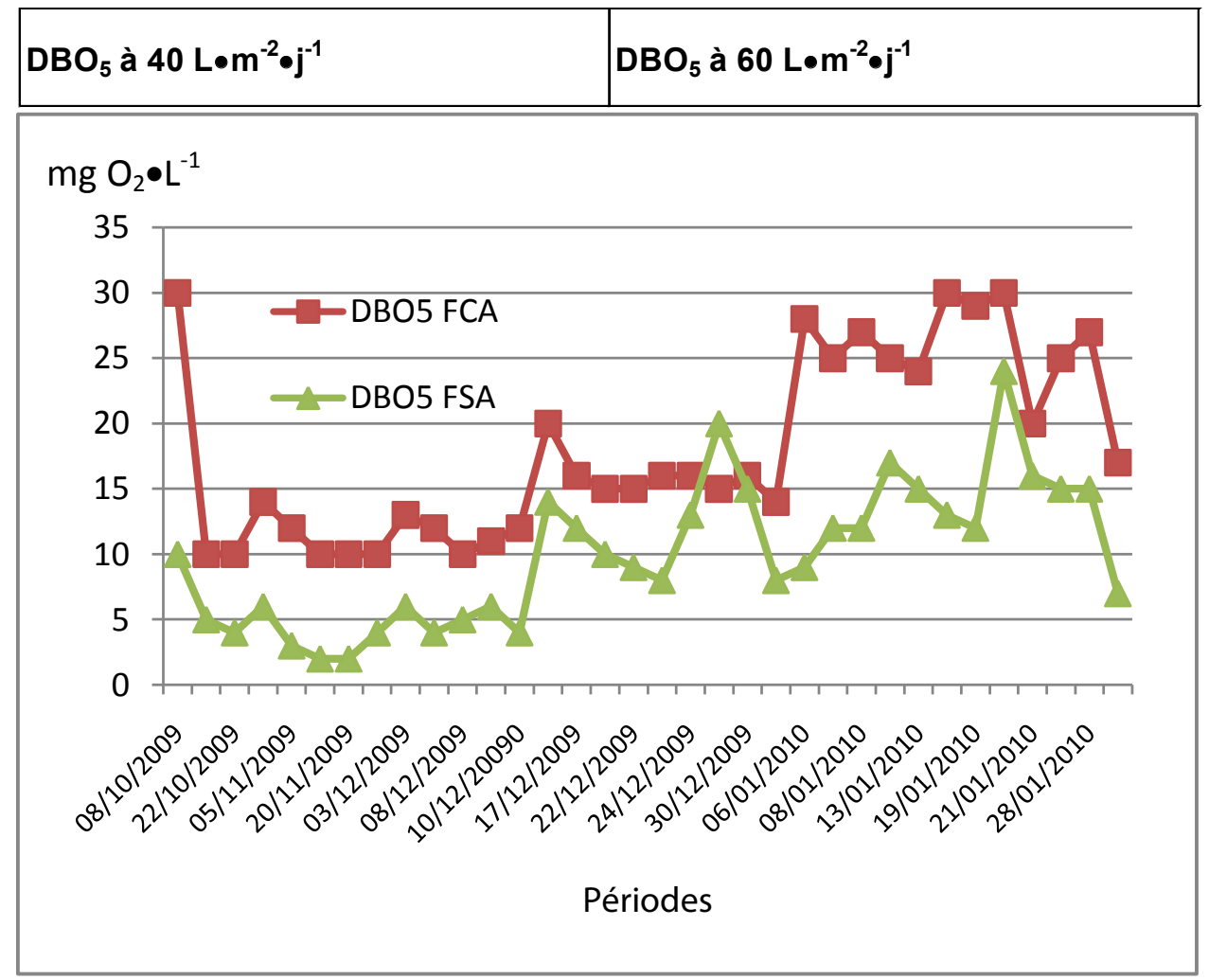

Figure 4. Évolution de la $\mathrm{DBO}_{5}$ des filtrats issus des pilotes filtre à sable aéré (FSA) et du filtre à

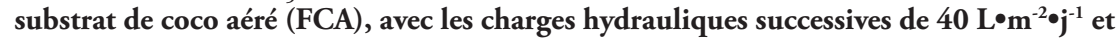
$60 \mathrm{~L} \cdot \mathrm{m}^{-2} \bullet \mathbf{j}^{-1}$.

Five-day biochemical oxygen demand (BOD $)_{5}$ removal for FSA and FCA with the two successive loads of $40 \mathrm{~L} \bullet \mathrm{m}^{-2} \cdot j^{-1}$ and $60 \mathrm{~L} \bullet \mathrm{m}^{-2} \bullet j^{-1}$.

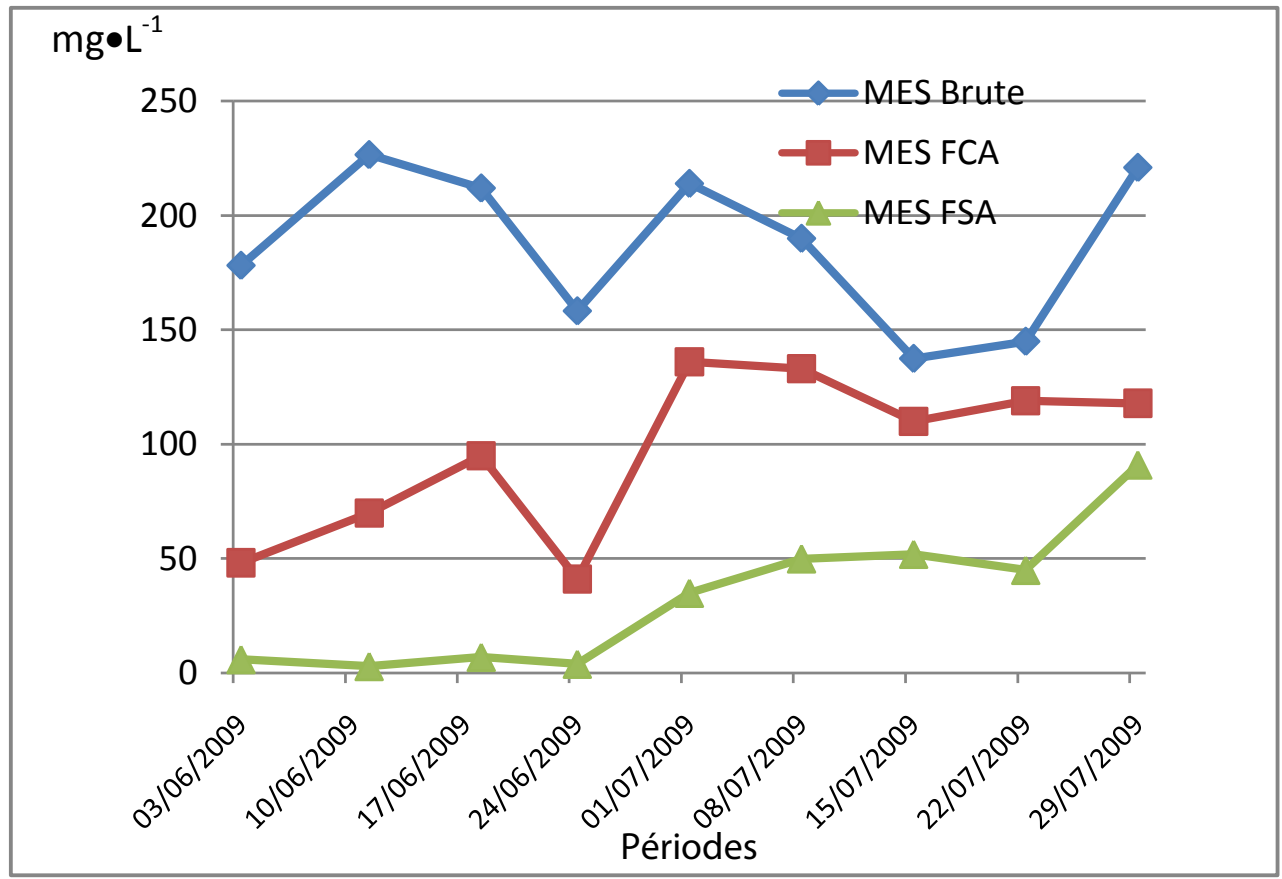

Figure 5. Évolution temporelle des teneurs en matières en suspension (MES) avec la charge

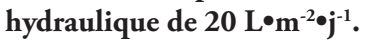

Suspended solids removal with the load of $20 \mathrm{~L}^{\circ} \mathrm{m}^{-2} \bullet \mathrm{j}^{-1}$. 


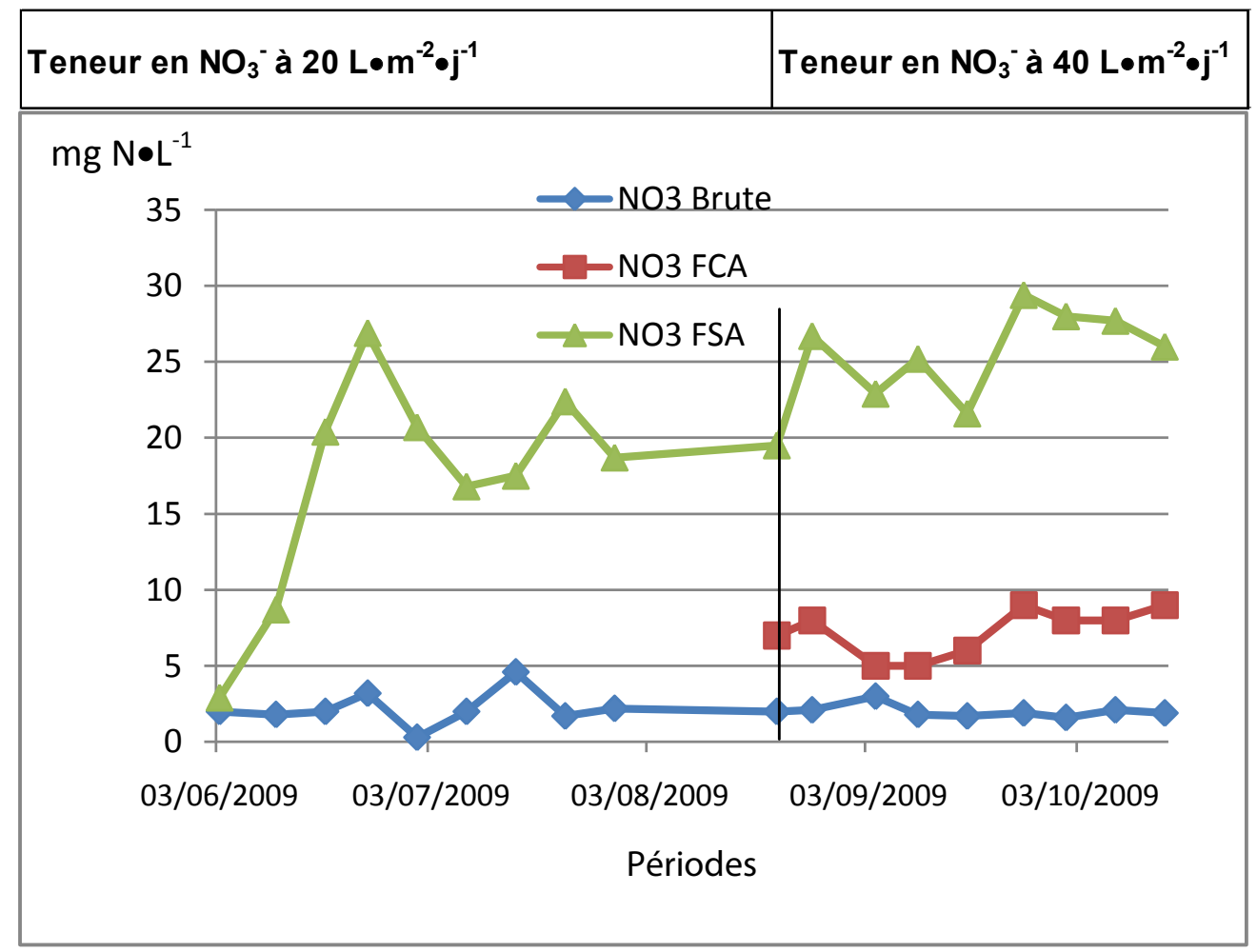

Figure 6. Variation temporelle de la concentration en nitrates pour les charges hydrauliques

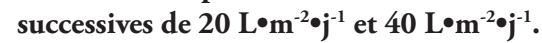

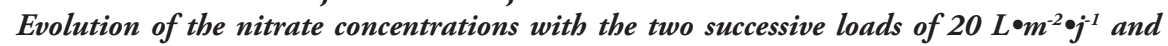
$40 \mathrm{~L} \cdot \mathrm{m}^{-2} \bullet j^{-1}$.

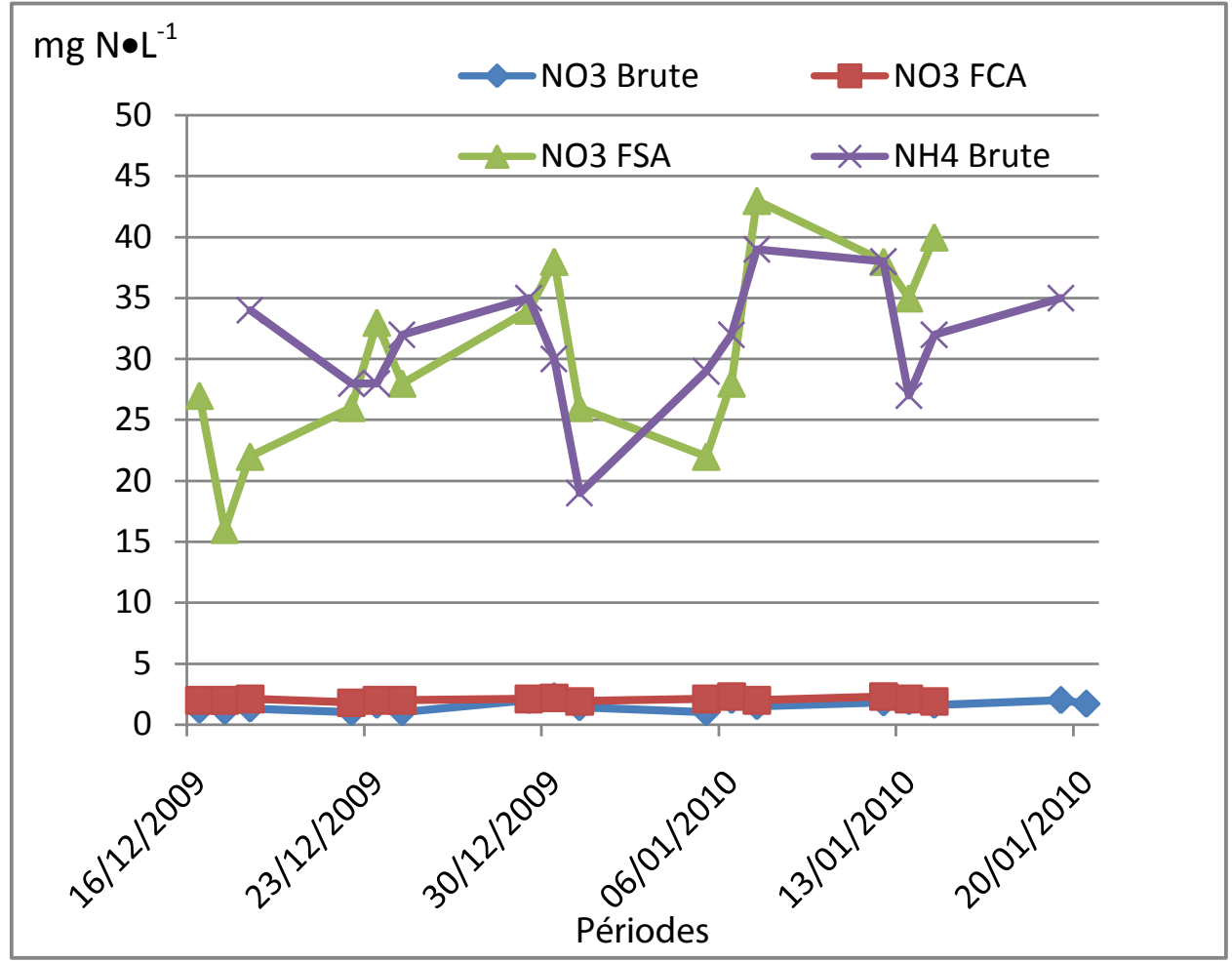

Figure 7. Variation temporelle de la concentration en nitrates pour la charge hydraulique maximale appliquée de $60 \mathrm{~L} \cdot \mathrm{m}^{-2} \bullet \mathbf{j}^{-1}$.

Evolution of the nitrate concentrations with the maximum hydraulic load of $60 \mathrm{~L}^{\bullet} \mathrm{m}^{-2} \bullet^{-1}$. 
un abattement de $84 \%$ donnant ainsi une teneur moyenne de $10 \mathrm{mg} \mathrm{N} \cdot \mathrm{L}^{-1}$, tandis que le rendement moyen de l'élimination de $\mathrm{NH}_{4}^{+}$est de $96 \%$ (Figure 8 ).

\section{DISCUSSION}

Les eaux résiduaires urbaines entrant à la station sont très biodégradables et cette caractéristique s'observe également à la sortie du bassin anaérobie où nous avons prélevé l'effluent d'alimentation des pilotes (Tableau 1). Ce bassin assure un temps de séjour de trois à cinq jours et permet un premier abattement de la $\mathrm{DBO}_{5}$ et des MES et un polissage des différents paramètres en général. En effet, dans le bassin anaérobie, à l'image d'autres procédés biologiques d'épuration des eaux usées, il se produit une combinaison de processus physiques, chimiques et biologiques. Une décantation des matières solides lourdes formant les boues au sein desquelles se développent des bactéries anaérobies. Ces bactéries assurent la fermentation et la solubilisation de la matière organique avec dégagement de $\mathrm{CH}_{4}, \mathrm{CO}_{2}$ et $\mathrm{H}_{2} \mathrm{~S}$ entre autres. Tout ceci a pour conséquence un abattement assez important de la DCO et des MES. L'épuration par les pilotes d'infiltration-percolation sur sable et sur substrat de coco a permis d'assurer des abattements supplémentaires sur ces différents paramètres de pollution (Tableau 3).

\subsection{Demande chimique en oxygène}

Avec la charge hydraulique de $20 \mathrm{~L} \cdot \mathrm{m}^{-2} \bullet \mathrm{j}^{-1}$, équivalant à des

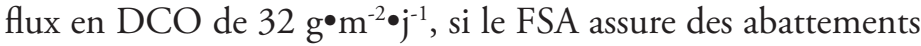
sur la DCO, avec un rendement moyen de $93 \%$, le FCA a donné un rendement moyen de $15 \%$ avec des rendements négatifs en début de fonctionnement (voir Figure 2). Cela pourrait s'expliquer par l'extraction de la matière du substrat de coco, dont le filtrat était très coloré malgré la macération et le lavage à l'eau claire pendant un mois. Il y a donc de la DCO ajoutée qui occulte l'abattement réel sur la pollution de l'effluent d'alimentation. En effet, NOGUERA (1998), cité par MOREL et al. (2000), rapporte que les fibres de coco ont une composition moyenne de 35 à $54 \%$ de lignine, 23 à $43 \%$ de cellulose et 3 à $12 \%$ d'hémicellulose, avec des composés phénoliques.

En outre, le substrat de coco présente une grande porosité qui est comblée par l'air ou l'eau en fonction de sa granulométrie (MOREL et al., 2000), ce qui favorise une percolation plus rapide de l'effluent comme nous avons pu l'observer lors des expériences (Tableau 2). WANKO et al. (2006) confirment cet

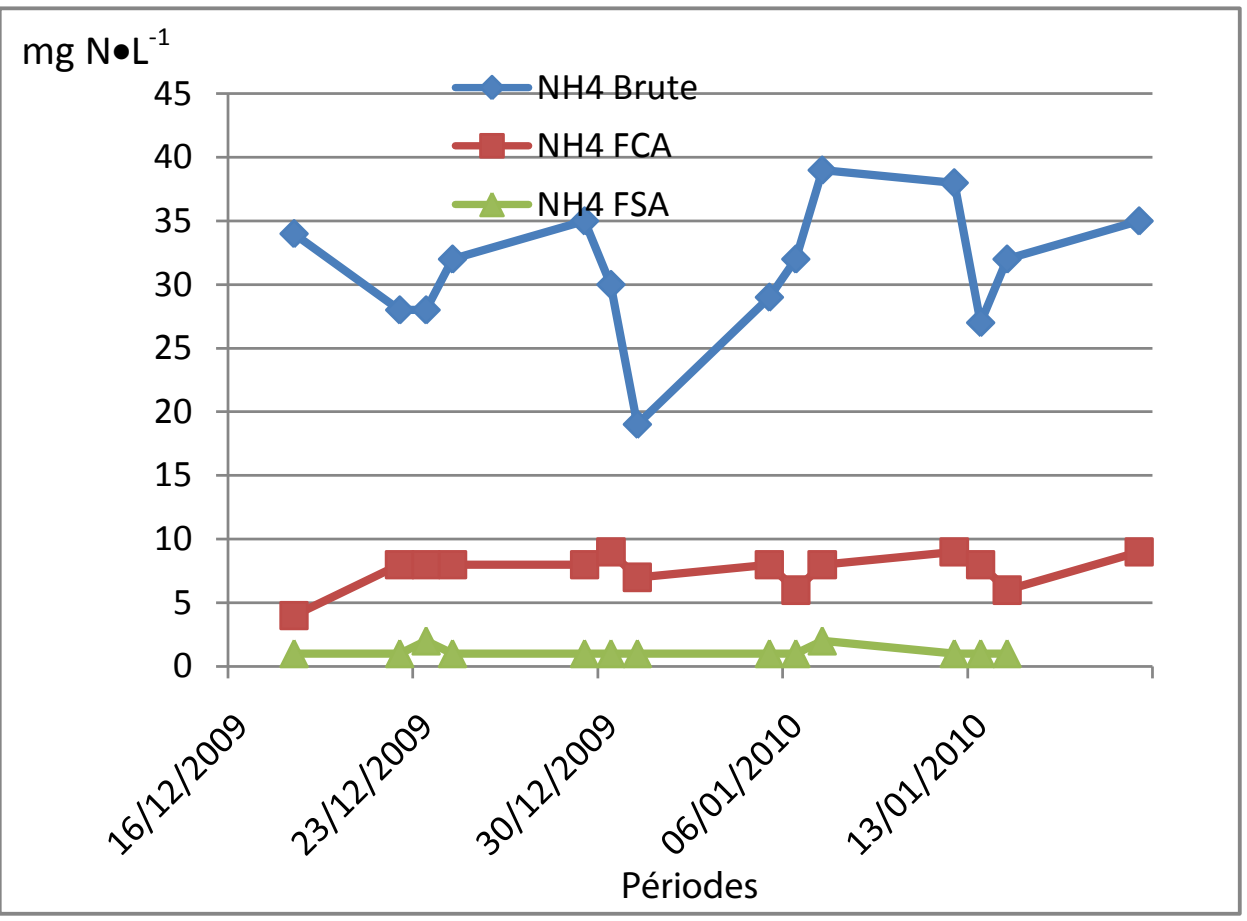

Figure 8. Variation temporelle de la concentration en ammonium pour la charge hydraulique de

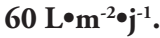

Evolution of the ammonium concentrations with the maximum hydraulic load of $60 \mathrm{~L} \cdot \mathrm{m}^{-2} \bullet j^{-1}$. 
Tableau 3. Valeurs moyennes et écarts-types des paramètres mesurés sur les filtrats issus du filtre à sable aéré (FSA) et du

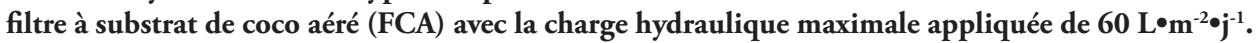

Table 3. Mean values and standard deviations of the parameters measured with FSA and FCA for maximal bydraulic load $\left(60 L \cdot m^{-2} \bullet j^{-1}\right)$.

\begin{tabular}{|c|c|c|c|c|c|c|c|}
\hline \multirow[b]{2}{*}{ Paramètres } & \multirow[b]{2}{*}{$n$} & \multicolumn{3}{|c|}{ FSA } & \multicolumn{3}{|c|}{ FCA } \\
\hline & & Moyenne & E.T. & $\begin{array}{c}\text { Enlèvement } \\
(\%)\end{array}$ & Moyenne & E.T. & $\begin{array}{c}\text { Enlèvement } \\
(\%)\end{array}$ \\
\hline $\mathrm{DCO}\left(\mathrm{mg} \mathrm{O}_{2} \bullet \mathrm{L}^{-1}\right)$ & 21 & 61 & 21 & 95 & 627 & 63 & 51 \\
\hline $\mathrm{DBO}_{5}\left(\mathrm{mg} \mathrm{O}_{2} \bullet \mathrm{L}^{-1}\right)$ & 21 & 13 & 4 & 98 & 21 & 6 & 98 \\
\hline $\mathrm{N}-\mathrm{NO}_{3}\left(\mathrm{mg} \bullet \mathrm{L}^{-1}\right)$ & 15 & 30 & 8 & & 2,0 & 0,1 & \\
\hline $\mathrm{N}-\mathrm{NH}_{4}\left(\mathrm{mg} \bullet \mathrm{L}^{-1}\right)$ & 15 & 1,15 & 0,37 & 96 & 7,57 & 1,39 & 75 \\
\hline $\mathrm{N}-\mathrm{NTK}\left(\mathrm{mg} \bullet \mathrm{L}^{-1}\right)$ & 10 & 10 & 6 & 84 & 16 & 3 & 75 \\
\hline
\end{tabular}

état de fait en dégageant une corrélation entre temps de contact effluent-biomasse et la dispersion de l'effluent au sein du massif. La dégradation de la pollution est d'autant plus efficace que le coefficient de dispersion est faible.

Par ailleurs, il faut noter également que cette phase correspond à la mise en place progressive du biofilm épurateur, qui doit assurer l'élimination de la pollution biodégradable.

La performance du FSA pourrait s'expliquer, d'une part, par le rôle de barrière physique (OUATTARA et al., 2008) assurée par le sable de granulométrie moyenne avec des pores plus réduits et, d'autre part, par une plus faible vitesse de percolation, donc un temps plus important de contact entre les eaux usées et la microflore épuratrice qui colonise progressivement le massif. Les travaux de KOUAME et al. (2008) confirment les performances épuratoires du sable de granulométrie moyenne en traitement des eaux usées et matières de vidange par infiltration-percolation.

Lorsque l'on passe au débit de $40 \mathrm{~L} \cdot \mathrm{m}^{-2} \bullet \mathrm{j}^{-1}$, on constate une amélioration notable des performances du FCA, avec un rendement moyen de $40 \%$, alors que le FSA présente un rendement épuratoire moyen de $95 \%$. Cependant, il est important de noter que le rendement reste positif durant toute la période d'alimentation à cette charge hydraulique. Le passage à une charge hydraulique d'alimentation de $60 \mathrm{~L} \cdot \mathrm{m}^{-2} \bullet \mathrm{j}^{-1}$ se traduit par une amélioration des rendements épuratoires du FCA qui atteint désormais une moyenne de $51 \%$ contre $95 \%$ pour le FSA. Cette amélioration notable des rendements d'élimination de la DCO avec le substrat de coco pourrait s'expliquer par le fait que la vitesse de percolation ayant augmenté avec l'augmentation des charges hydrauliques, il y a une plus grande dilution des substances extraites (composés phénoliques, dont les tanins) et une diminution progressive de leur extraction par épuisement des " réserves » contenues dans le substrat de coco, ceci d'autant plus que, parallèlement, nous avons observé que le filtrat devenait de plus en plus clair avec le temps. À cela, on pourrait ajouter la colonisation de plus en plus importante du massif filtrant par la biomasse épuratrice, ce qui contribuerait à l'amélioration des rendements. La poursuite des essais sur une période plus longue pourrait permettre d'atteindre des rendements plus importants avec le FCA. Cette hypothèse est confortée par les résultats des travaux de (LEE et al., 2007) faisant état des rendements de $80 \%$ sur la DCO par utilisation de fibres de coco dans le traitement des eaux usées de porcherie.

\subsection{Demande biochimique en oxygène}

La $\mathrm{DBO}_{5}$ est relative à la portion biodégradable des eaux usées, c'est-à-dire oxydable par des bactéries. Les échantillons d'eau traitée issue du FSA et du FCA ont été ensemencés avec un prélèvement issu du bassin anaérobie du jour avant la mise à l'étuve à $20^{\circ} \mathrm{C}$. Comme le montre la figure 4 , aussi bien le FSA que le FCA assurent un excellent abattement sur la $\mathrm{DBO}_{5}$ avec des rendements respectifs 99 et $98 \%$, confirmant une bonne colonisation des deux massifs filtrants par les microorganismes, mais également la bonne aération des massifs favorisée par l'alternance de phases. En effet, l'alternance de phases favorise une autorégulation du biofilm et une recharge en oxygène des milieux granulaires (GNAGNE et BRISSAUD, 2002; MENORET, 2001).

Selon RENMAN et al. (2008) qui ont testé les capacités de traitement de six matériaux granulaires, dont le sable, et noté des rendements d'au moins $97 \%$ sur la $\mathrm{DBO}_{5}$ avec l'ensemble de ces matériaux, ces performances relèvent en partie de l'efficacité du prétraitement qui permet d'éviter le colmatage.

Quand on passe à une charge hydraulique plus élevée, les deux matériaux conservent pratiquement le même niveau de performance.

En somme, si sur la DCO, le FCA a donné des rendements peu satisfaisants du fait de l'extraction de composés phénoliques, surtout au début de son utilisation, ces performances se sont améliorées avec le temps. En revanche, sur l'abattement de la pollution biodégradable $\left(\mathrm{DBO}_{5}\right)$, ce matériau de filtration se 
montre presqu'aussi performant que le sable moyen, considéré par KOUAME et al. (2008) comme présentant les meilleures conditions de filtration, surtout quand il s'agit d'effluents chargés.

\subsection{Matières en suspension}

Les rendements épuratoires sur les MES mesurés pour des charges hydrauliques de $20 \mathrm{~L} \cdot \mathrm{m}^{-2} \bullet \mathrm{j}^{-1}$ sont plus importants pour le FSA comparativement à ceux obtenus avec le FCA pour des moyennes respectives de $82 \%$ et $47 \%$. Cela est prévisible, étant donné l'extraction de la matière par la percolation de l'ERU à travers le FCA.

Cependant, on note une baisse des rendements au niveau des deux substrats liée à la prolifération progressive des bactéries formant un biofilm au sein du massif, réduisant ainsi la porosité du milieu granulaire. La phase de repos favorise une réduction de la croissance bactérienne liée à une famine (WANKO et al., 2005) et un stress hydrique avec, pour conséquence, un détachement d'une partie du biofilm qui passe dans le filtrat, ceci d'autant plus que nous n'avons pas observé de colmatage pendant les neuf mois de fonctionnement sans interruption. Ces résultats sont confortés par les conclusions d'autres travaux (COULIBALY et al., 2008; OUATTARA et al., 2008; YEO et al., 2008).

\subsection{Azote}

Le processus d'élimination de l'azote est amorcé par la nitrification. Le FSA présente un taux de nitrification qui reste croissant avec les trois charges hydrauliques successives, pour des concentrations en $\mathrm{N}-\mathrm{NO}_{3}$ atteignant $27 \mathrm{mg} \bullet \mathrm{L}^{-1}$ à la charge de $20 \mathrm{~L} \bullet \mathrm{m}^{-2} \bullet \mathrm{j}^{-1}, 30 \mathrm{mg} \bullet \mathrm{L}^{-1}$ avec la seconde charge hydraulique (Figure 6) et $40 \mathrm{mg} \bullet \mathrm{L}^{-1}$ à $60 \mathrm{~L} \cdot \mathrm{m}^{-2} \bullet \mathrm{j}^{-1}$ (Figure 7), et ce, pour des teneurs moyennes respectives en nitrates et NTK de $2 \mathrm{mg} \mathrm{N}-\mathrm{NO}_{3} \cdot \mathrm{L}^{-1}$ et $63 \mathrm{mg} \mathrm{N} \bullet \mathrm{L}^{-1}$ dans l'effluent brut.

Le FCA nitrifie très peu avec des concentrations moyennes en $\mathrm{N}-\mathrm{NO}_{3}$ de $7 \mathrm{mg} \cdot \mathrm{L}^{-1}$, alors que FSA donne une teneur moyenne de $25 \mathrm{mg} \mathrm{N}-\mathrm{NO}_{3} \bullet \mathrm{L}^{-1}$. À $60 \mathrm{~L} \cdot \mathrm{m}^{-2} \bullet \mathrm{j}^{-1}$, le FCA s'est révélé encore moins performant avec des concentrations en nitrates à peine supérieures aux teneurs de l'effluent d'alimentation comme le montre la figure 7.

La bonne performance du FSA est sans doute inhérente à la bonne aération du massif, d'autant plus que nous n'avons pas observé d'effets de colmatage, grâce certainement à un temps de repos suffisant (quatre jours) pour assurer une autorégulation du biofilm et une recharge du massif en oxygène. Notons néanmoins que WANKO et al. (2005) ont obtenu des abattements plus importants de l'azote avec du sable concassé par rapport au sable alluvionnaire et un résultat inverse sur les rendements en DCO.

En dépit de la bonne oxygénation du milieu, compte tenu de la grande porosité du substrat et du temps de repos, le FCA présente une nitrification faible à pratiquement nulle quand on passe d'une charge hydraulique de 40 à $60 \mathrm{~L} \cdot \mathrm{m}^{-2} \bullet \mathrm{j}^{-1}$. D'une part, cela pourrait être dû à un temps de séjour hydraulique plus court, et au débit de percolation plus important de $25 \mathrm{~mL} \cdot \mathrm{min}^{-1}$ au sein du massif de substrat de coco contre $7 \mathrm{~mL} \cdot \mathrm{min}^{-1}$ pour le FSA pendant les trois premières heures de l'écoulement, ce qui se traduit par un écoulement plus rapide lié également à l'accroissement du débit d'alimentation. Les faibles teneurs en nitrates avec le substrat de coco pourraient également être inhérentes à sa richesse en composés phénoliques. En effet, selon MACHEIX et al. (2005), les tanins qui sont des composés phénoliques s'associent aux composés azotés en milieu aqueux en formant des complexes qui précipitent. Nous pensons donc que les tanins extraits du substrat de coco ont pu se combiner avec les composés azotés contenus dans l'effluent, provoquant ainsi leur précipitation, ce d'autant plus que malgré la faible nitrification du FCA, on observe des abattements de $75 \%$ respectivement de $\mathrm{NTK}$ et $\mathrm{NH}_{4}{ }^{+}$à l'issue de l'épuration de l'ERU par le FCA. Les concentrations en nitrites des filtrats restent quasiment nulles aussi bien au niveau du FCA que du FSA.

MACHEIX et al. (2005) expliquent également que les tanins ont une action inhibitrice sur certaines bactéries. La très faible nitrification au sein du massif de substrat de coco pourrait relever également de l'impact des tanins sur les bactéries nitrifiantes.

Par ailleurs, PELL et al. (1990), cités par RENMAN et al. (2008), ont noté une décroissance du nombre de bactéries quand on passe des couches supérieures du massif filtrant vers le fond, et ce phénomène est d'autant plus prononcé que le milieu est alcalin. BAHGAT et al. (1999) indiquent que les bactéries Nitrosomonas assurant l'oxydation de $\mathrm{NH}_{4}^{+}$en $\mathrm{NO}_{2}{ }^{-}$ sont plus abondantes que celles assurant l'oxydation des $\mathrm{NO}_{2}^{-}$ en $\mathrm{NO}_{3}$. Les observations ci-dessus permettent de déduire que les mécanismes intervenant dans l'élimination de la pollution ont lieu essentiellement dans la partie supérieure du massif (WANKO et al., 2006) et une percolation trop rapide aura pour conséquence une baisse des capacités de nitrification du substrat de coco.

\section{CONCLUSION}

Cet essai de traitement des ERU par infiltration-percolation sur sable et sur substrat de coco a permis de mettre en évidence les performances incontestées du sable en matière d'élimination 
de la pollution organique ( $95 \%$ de la DCO, $99 \%$ de la $\mathrm{DBO}_{5}$ et $82 \%$ des MES) et la pollution azotée, avec des effluents relativement chargés et une charge hydraulique maximale de $60 \mathrm{~L} \cdot \mathrm{m}^{-2} \cdot \mathrm{j}^{-1}$.

Quant au substrat de coco, ses performances sont bonnes en matière d'abattement de la pollution biodégradable (98\% de la $\mathrm{DBO}_{5}$ ). Concernant la DCO, un lessivage important du substrat s'impose afin d'extraire les composés phénoliques très peu biodégradables qui impactent négativement les performances du filtre sur ce paramètre. La nitrification semble être inhibée par les tanins qui formeraient des complexes solides avec les composés azotés contenus dans les eaux usées. Si les performances du substrat de coco s'améliorent avec l'épuisement progressif des composés phénoliques extraits, l'augmentation des charges hydrauliques a pour conséquence une percolation plus rapide de l'effluent à travers le substrat de coco, donc un temps de contact moins long avec les microorganismes. Cet état de fait nuit également à la nitrification. Une alimentation à faibles charges hydrauliques pour des effluents de fortes charges organiques constituerait un gage de meilleurs rendements épuratoires par une optimisation des conditions d'oxygénation des massifs. L'alternance de phases d'alimentation et de phases de repos observée dans le fonctionnement des pilotes et la répartition en deux bâchées a permis d'avoir des résultats satisfaisants sur l'abattement de la pollution organique et la nitrification avec le sable, mais seulement sur la pollution organique avec le substrat de coco. Un temps de repos moins long pourrait être compatible avec des charges hydrauliques inférieures à $60 \mathrm{~L} \cdot \mathrm{m}^{-2} \bullet \mathrm{j}^{-1}$, pour des charges organiques aussi importantes que celles de l'effluent utilisé.

\section{REMERCIEMENTS}

Les auteurs remercient la Fondation Internationale pour la Science (IFS) pour le soutien financier apporté, la Direction de l'Institut du Génie de l'Environnement et Écodéveloppement de l'Université Claude-Bernard Lyon 1 (France), la Direction Générale et le personnel du laboratoire de l'Office National de l'Eau et de l'Assainissement (ONEA) du Burkina Faso, pour leur appui et leurs contributions à l'analyse des échantillons.

\section{RÉFÉRENCES BIBLIOGRAPHIQUES}

BAHGAT M., A. DEWEDAR et A. ZAYED (1999). Sand filters used for wastewater treatment: buildup and distribution of microorganisms. Water Res., 33, 1949-1955.
COULIBALY L., B.A. ASSAMOI, I. SAVANE et Y. BADO

(2008). Un filtre à sable perforé à alimentation intermittent pour l'épuration des effluents d'huilerie de palme : Cas de l'unité industrielle de Toumanguié, Côte d'Ivoire. Eur. J. Sci. Res., 20, 226-238.

FALIENOR (2006). Substrats. La lettre d'information FalienorTerreaux de France, 17, 2-4.

GNAGNE T. et F. BRISSAUD (2003). Étude des potentialités d'épuration d'effluents d'abattoir par infiltration sur sable en milieu tropical. Sud Sci. Technol., 11, 4-11.

GNAGNE T. et F. BRISSAUD (2002). Étude des limites d'épuration par infiltration sur sable des eaux usées fortement chargées en matières oxydables de l'abattoir de Ouagadougou au Burkina Faso. Sud Sci. Technol., 9, 34-41.

KONE M., Y. BOUVET, L. BONOU, J. KOULIDIATI et P. JOLY (2009). Étude de la pollution des eaux par les intrants agricoles: Cas de cinq zones d'agriculture intensive du Burkina Faso. Sud Sci. Technol., 17, 6-15.

KOUAME Y. F., T. GNAGNE et K.F. KONAN (2008). Choix du matériau poreux pour l'épuration des boues de vidange des fosses septiques par lit de séchage à écoulement non saturé (LSENS). Trib. Eau, 642, 15-24.

LEE S., H. LEE, S. LEE et S. CHITAPORNPAN (2007). Media configuration and recirculation of upflow anaerobic floating filter for piggery wastewater treatment. Korean J. Chem. Eng., 24, 980-988.

MACHEIX J.J., A. FLEURIET et C. J. ALLEMAND (2005). Les composés phénoliques des végétaux : Un exemple de métabolites secondaires d'importance économique. Presses Polytech. Univ. Romandes, 192 p.

MENORET C. (2001). Traitement d'effluents concentrés par cultures fixées sur gravier ou pouzzolane. Thèse de Doctorat, Univ. Montpellier II, France, 130 p.

MOREL Ph., L. PONCET et L.M. RIVIÈRE (2000). Les supports de culture horticoles. INRA Editions, $87 \mathrm{p}$.

NOGUERA P. (1998). Communication personnelle, cité dans MOREL Ph., L. PONCET et L.M. RIVIÈRE (2000). Les supports de culture horticoles. INRA Editions $87 \mathrm{p}$.

OUATTARA P.J.M., L. COULIBALY, P.N. MANIZAN et G. GOURENE (2008). Traitement des eaux résiduaires urbaines par un marais artificiel à drainage vertical planté avec Panicum Maximum sous climat tropical. Eur. J. Sci. Res., 23, 25-40. 
PELL, M., F. NYBERG et H. LJUNGGREN (1990). Microbial numbers and activity during infiltration of septic-tank effluent in a subsurface sand filter. Water Res., 24, 1347-1354.

RENMAN A., L.D. HYLANDER et G. RENMAN (2008). Transformation and removal of nitrogen in reactive bed filter materials designed for on-site wastewater treatment. Ecol. Eng., 34, 207-214.

TILLEY E., C. LÜTHI, A. MOREL, C. ZURBRÜGG et R. SCHERTENLEIB (2008). Compendium of sanitation systems and technologies. Swiss Federal Institute of Aquatic Science and Technology (Eawag). Dübendorf, Switzerland. $157 \mathrm{p}$.

WANKO A., R. ROSE et A. LIENARD (2005). Capacities of infiltration-percolation processes for the treatment of a synthetic effluent. Rev. Sci. Eau, 18, 165-175.

WANKO A., R. MOSE et A. SADOWSKI (2006). Simulation des transferts réactifs multi-constituants au sein des lits d'infiltration-percolation - évaluation des capacités d'oxygénation. Rev. Sci. Eau, 19, 199-212.

WETHE J., M. RADOUX et E. TANAWA (2003). Assainissement des eaux usées et risques socio-sanitaires et environnementaux en zones d'habitat planifié de Yaoundé (Cameroun). VertigO - Rev. Sci. Environ., 4. 1-12.

YEO T.M., T. GNAGNE, K.F. KONAN, Y.F. KOUAME et P.V. HOUENOU (2008). Étude de la vulnérabilité d'un lit de séchage d'effluents de fosses septiques à écoulement non saturé. Eur. J. Sci. Res., 24, 187-196. 